\title{
How Can We Make Industry Adopt Formal Methods?
}

\author{
Takuya Katayama \\ Japan Advanced Institute of Science and Technologies
}

Despite a long history of technical development of formal methods and their success in advanced system developments, they are not well recognized nor accepted as effective and standard methodology in industrial and commercial software development. This fact should be considered seriously if we evaluate their intrinsic technical superiority. Though ignorance of the new technologies will be one reason for the reluctance of industry to use formal methods, we should also check if current formal methods will be enough for the real software development practices in industry. In this talk, based on interviews with software engineers and managers, these issues will be addressed including proposal of formal methods adoptable in industry. 